\title{
La formación en valores: algunas ideas a modo de ensayo
}

\section{GATTAS ABUGATTAS*}

Esta presentación se encuentra dividida en tres partes. En las dos primeras se intentará responder a las siguientes preguntas: ¿qué entendemos por valores? y icómo formar en valores en el ámbito del Derecho? En una tercera parte dejaré planteado un problema que, a raíz de la explicación previa que haré, me parece que surge respecto de la formación en valores.

\section{LOS VALORES}

Normalmente, cuando hablamos de valores, solemos hacer referencia a la libertad, a la justicia, a la verdad, entre otros. Así, decimos que «los valores son la libertad, la justicia...». Sin embargo, si nos fijamos con un poco más de detalle en esto, notaremos que al hablar de los valores lo usual es hacer referencia a «cuáles son» y no propiamente a «qué son».

En una definición bastante permisiva, los valores pueden ser descritos como «ideas», «comportamientos esperados» o incluso «cosas» que son positivamente apreciadas por el ser humano. Por ejemplo, podemos decir que la libertad es una idea muy apreciada; la justicia, entendida como el comportamiento justo de las personas, suele ser algo deseado, esperado; el oro es un bien querido por muchos, en tanto que su tenencia es apreciada por los seres humanos. De esta descripción se desprenden varios temas.

\section{I.1. El que valora es el ser humano}

En efecto, a decir del profesor Carlos Fernández Sessarego, el ser humano es una unidad psicosomática sustentada en su libertad. Es decir, las personas no «tienen» libertad, sino que «son» libertad. Encontramos un ejemplo de esto en nuestro himno nacional, cuya letra expresa «Somos libres. Seámoslo siempre» y no «Tenemos libertad. Mantengámosla siempre».

Además, el ser humano es un ser social que se desarrolla en la historia, que cambia, que se adapta, que evoluciona. Aquello que en un momento de su historia es apreciado puede dejar de serlo después. Solo así se puede entender que los valores evolucionen junto con el ser humano que valora. De tal modo, dichos valores no son estáticos,

* Es profesor de Derecho en pregrado y postgrado en la Pontificia Universidad Católica del Perú. El presente artículo es una adaptación de la ponencia presentada durante el Seminario Internacional sobre Enseñanza del Derecho, desarrollado en la Facultad de Derecho de la PUCP entre el 9 y el 11 de agosto de 2010. 
sino dinámicos. Por ejemplo, la esclavitud ha pasado de ser considerada normal a ser vista como algo reprochable.

Resulta entonces que esta unidad psicosomática libre y social, poniendo en práctica la libertad que «es», asigna valor a lo que la rodea; es decir, valora. De esto se desprende algo muy sencillo: valorar es simplemente el acto mediante el cual el ser humano asigna valor. Un ejemplo muy citado por el profesor Fernández Sessarego es el de la historia bíblica que narra la muerte de Abel en manos de su hermano Caín. Ante este hecho se presume la valoración de la madre de ambos, Eva, quien seguramente lloró luego de ocurrido este hecho, lo que denotaría una valoración negativa del asesinato y, consecuentemente, una valoración positiva de la vida.

\subsection{Valoramos lo que nos rodea}

El ser humano, se ha explicado, es quien asigna valor, es decir, quien valora. $\mathrm{Al}$ ser el ser humano social por naturaleza, resulta que será coexistencial. Las personas conviven entre sí en un espacio y en un tiempo determinados; por lo tanto, valorarán los elementos que se relacionan con esa convivencia, en ese espacio y en ese tiempo. Es decir, valorarán un conjunto de aspectos materiales e inmateriales relacionados con su vida misma.

\section{I.3. En el ámbito del Derecho}

Normalmente, en el ámbito del Derecho asignamos valor positivo a ciertas ideas, pero también a las cosas y a los objetos, por ejemplo, a los bienes de las personas. Sin embargo, si nos enfocamos en lo inmaterial, solemos asignar valor:

- a la idea de libertad, porque nos entendemos libres (idea que ha cambiado en el tiempo);

- a la idea de justicia, porque creemos que ella facilita nuestra coexistencia;

- a la verdad, porque no queremos vivir rodeados de mentira; y

- a muchos otros «valores» que son útiles, pues nos permiten entender y juzgar nuestra realidad.

En suma, las personas valoramos todo aquello que rodea al conjunto de relaciones intersubjetivas que forman la vida del ser humano, y también valoramos esas mismas relaciones. Esos valores, luego, son plasmados algunas veces en normas morales o éticas y, otras, en normas jurídicas. Este es el origen tridimensional del Derecho: la interacción entre un conjunto de relaciones intersubjetivas, la valoración de esas relaciones y las normas jurídicas que recogen esa valoración ${ }^{1}$. 


\section{LA FORMACIÓN EN VALORES}

La manera en que se lleve a cabo la formación en valores deberá considerar varios aspectos. Entre ellos, me enfocaré en cuatro. En primer lugar, la formación en valores dependerá de aquello que sea valorado. Podemos tener valores como seres humanos (la vida), valores como peruanos (los símbolos patrios), valores como abogados (la justicia). La formación en cada uno de estos «rubros» de valores debe ser distinta.

En segundo lugar, si nos ubicamos dentro del rubro de valores como abogados, tendremos que ponernos de acuerdo sobre cuáles son los valores que debe tener un abogado. Aquí surge un primer problema: no todos valoramos igual. Esto responde a la dignidad misma del ser humano. Todos somos iguales en tanto que todos somos libres; sin embargo, todos somos distintos en tanto que, en ejercicio de nuestra libertad dentro del bien común, cada uno hace con ella lo que quiere. Entiendo la dignidad, entonces, como la condición que posee todo ser humano de ser igual y a la vez distinto de los demás, condición que se traduce en la exigencia de respecto, entendido como la consideración a los otros y a sus ideas.

El hecho es, entonces, que no todos valoramos de la misma manera aquello que nos rodea. Por ejemplo, no es igual el valor que las personas le dan a la idea de éxito profesional. Para algunos es cuestión de dinero; para otros es cuestión de realización personal. Esto, en el ámbito del Derecho, es muy importante, pues en función de lo que entendamos por éxito profesional tomaremos un conjunto de decisiones que pueden ser entendidas como éticamente correctas o incorrectas. De este modo, si los estudiantes de Derecho consideran que el éxito profesional se mide por el dinero que ganan, es muy probable que, precisamente para ganarlo, estén dispuestos a ir más allá de la ética del abogado.

En tercer lugar, suponiendo que haya un acuerdo sobre aquellos valores que debe tener un abogado, o por lo menos sobre algunos de esos valores, considero que existen dos principales maneras para formar en ellos. Esto, preciso, lo planteo no como pedagogo especialista en formación en valores sino simplemente como profesor de Derecho.

Las dos principales maneras para formar en valores son (a) mostrando a los estudiantes qué pasaría si no existieran tales valores y (b), lo que es obvio, con el ejemplo.

\section{II.1. ¿Qué pasaría si los abogados no tuvieran los valores que se considera que deben tener?}

Lo que propongo en este punto es mostrar un mundo de injusticias para enseñar el valor de la justicia y mostrar un mundo de engaño y mentira para enseñar el valor de la verdad. Parto de la idea bastante aceptada de que «uno quiere lo que no tiene».

LA FORMACIÓN EN VALORES:

ALgUNAS IDEAS A MODO DE ENSAYO 
Por ejemplo, algunos profesores citan casos de negociación en los que el engaño aparenta traer mayores ganancias, pero al final del ejercicio queda demostrado que el engaño termina por producir resultados subóptimos.

Los elevados niveles de injusticia, de mentira y de corrupción que existen en el Perú y que de una u otra manera nos afectan facilitan, paradójicamente, la formación en valores siguiendo esta técnica. Es necesario mostrarle al estudiante que la situación se puede revertir, lo que es algo ciertamente difícil, aunque es una labor que está en sus manos.

\section{II.2. Lo obvio: enseñar con el ejemplo}

Esto es bastante conocido y algunas veces resulta tan obvio que lo dejamos de lado. Sin embargo, nadie puede negar que la mejor manera de enseñar a ser un «buen abogado» (entiéndase uno con valores) es siendo nosotros mismos, como profesores, buenos abogados.

Uno enseña a no mentir cuando no miente, a no estafar cuando no estafa, a no sobornar cuando no soborna. No obstante, aquí surge un segundo problema: en el Perú esto es muy difícil. Sería ingenuo de nuestra parte negar la realidad de nuestro país, en la que la gran mayoría de abogados alguna vez tuvo que mentir o, incluso, que sobornar para ganar un juicio, pese a que, muy probablemente, en esa ocasión la justicia haya estado de su lado.

Así, lo que no puede ocurrir es que un profesor de Derecho tenga un discurso en clase y en el mundo se comporte de manera completamente distinta. No puede existir una disociación tal que lleve a que en un contexto se diga algo y, en el otro, difícilmente se cumpla lo dicho.

En cuarto lugar, a estas dos maneras de formar en valores se les puede adornar con un conjunto de herramientas metodológicas muy diversas. Por ejemplo, se puede recurrir al cine, a casos reales, a casos especialmente diseñados sobre la base de concepciones teóricas que provengan de otras áreas del saber (como la economía, la ciencia política, las matemáticas, entre otras).

Enfrentar al estudiante a dilemas éticos durante la etapa de su formación representa otra herramienta metodológica útil. Sobre la base de la tolerancia, se podrá discutir en clase los problemas planteados, y esto deberá hacerse de la manera más abierta posible. Ello implica, por cierto, no suponer nada: como profesores, no podemos considerar que nuestros estudiantes poseen una formación básica en valores que viene de sus familias o de sus colegios. El contexto que envuelve a cada estudiante es muy diverso.

Otro tema en el que se insistiría está referido a la idea del éxito profesional. Muchos estudian Derecho porque creen que es la panacea que los hará millonarios, idea que no solo se aleja de la verdad en la mayoría de casos sino que, además, por lo menos merece ser discutida. Es necesario, 
considero, hacer notar a los estudiantes de Derecho la importancia de la persona y de su realización como tal. El Derecho es una ciencia social y su objeto final es el ser humano, servir a las personas. Una mala concepción del éxito profesional puede conducir a la toma de decisiones incorrectas frente a los dilemas éticos que los estudiantes enfrentan desde su etapa como practicantes y que seguirán enfrentando durante su vida profesional.

En suma, tendremos que ponernos de acuerdo sobre cuáles son los valores que queremos que hagan suyos nuestros estudiantes. Solo después de hacerlo podremos, de diferentes maneras y con muy diversas herramientas metodológicas, que cada vez deben ser más originales y fáciles de aceptar por los estudiantes, conducir una adecuada formación en valores.

\section{LA FORMACIÓN EN VALORES Y LA EDUCACIÓN VIRTUAL}

La pregunta que me hago luego de todo lo antes dicho es icómo formar en valores en un modelo de educación virtual? Debo confesar que aún no he encontrado una respuesta a esta pregunta, si es que la hubiere.

Parto de la premisa de que, durante el pregrado, el contacto humano es fundamental y es precisamente ese contacto el que contribuye a la formación en valores. Me refiero, en concreto, a la enseñanza de valores a través de ejemplos de vida.

En la universidad, el estudiante aprende a convivir como adulto con otros adultos, a comportarse de una manera determinada frente a gente que piensa distinto, tanto dentro como fuera de las aulas. Además, en un mundo tan visual como el actual, uno aprende a ser un abogado con valores viendo a otros abogados con valores y analizando su comportamiento. Aún no estoy convencido de que la educación virtual, que no permite ese contacto humano, mas solo a través de una interfaz digital, pueda estar capacitada para ser una herramienta útil en la formación en valores.

Parto de la hipótesis de que la educación virtual es muy importante y útil en niveles de especialización como maestrías, diplomaturas, cursos de actualización, entre otros similares. Sin embargo, considero que la educación virtual no debe ser empleada durante el pregrado, salvo de manera muy eventual o limitada a una cantidad determinada de cursos que, además, por sus propias exigencias metodológicas, puedan desarrollarse mejor de manera virtual.

Pero reitero que esta es solo una hipótesis. Por ello, lo que pretendo en este punto es simplemente dejar planteado el tema y proponer dos preguntas que lleven a una discusión posterior: icómo formar en valores en un modelo de educación virtual? y ise puede? 